\title{
PAdRões de distribuição GEOGRÁFICA dAS ESPÉCIES BRASILEIRAS de Pfaffia (Amaranthaceae) ${ }^{1}$
}

\author{
Maria Salete Marchioretto ${ }^{2,5}$, Silvia Teresinha Sfoggia Miotto \\ \& Josafá Carlos de Siqueira ${ }^{4}$
}

\begin{abstract}
Resumo
(Padrões de distribuição geográfica das espécies brasileiras de Pfaffia (Amaranthaceae)) O gênero Pfaffia Mart. é neotropical, sendo o Brasil considerado o centro de diversidade com 20 espécies, das quais 19 ocorrem na província biogeográfica do Cerrado. Suas espécies apresentam padrões de distribuição geográfica desde amplos até endêmicos. As províncias mais pobres em número de espécies foram a Amazônica e a Pampeana com três espécies em cada uma. $\mathrm{O}$ estado de Minas Gerais pode ser considerado o centro de diversidade e de endemismo do gênero, com espécies ocorrendo principalmente nos cerrados e campos rupestres.

Palavras-chave: diversidade, padrões de distribuição, cerrado, Minas Gerais, campos rupestres.
\end{abstract}

\section{Abstract}

(Patterns of geographic distribution of the Brazilian species of Pfaffia (Amaranthaceae)) The Brazil constitutes the main diversity center of the neotropical genus Pfaffia, with ca. 20 species, of which 19 occur in the "Cerrado" biogeographic province. Their species present distribution patterns ranging from wide to endemic. The poorest provinces in number of species are the "Amazônica" and "Pampeana" with three species each. The State of Minas Gerais can be considered the center of diversity and endemism with species occurring mainly in the savanna-like "cerrados" and rocky grasslands of the "campos rupestres".

Key words: diversity, distribution patterns, cerrado, Minas Gerais, campos rupestres.

\section{INTRODUÇÃO}

Amaranthaceae Juss. é considerada uma família de distribuição tropical e subtropical, sendo encontrada principalmente nas Américas e na África (Siqueira 2004). A família pertence à ordem Caryophyllales e, segundo características morfológicas (Judd et al. 2002) e moleculares (APG II 2003), inclui as Chenopodiaceae, totalizando 169 gêneros e 2360 espécies. Para Souza \& Lorenzi (2008) as Amaranthaceae apresentam 170 gêneros e 2000 espécies, sendo que no Brasil ocorrem 20 gêneros nativos com aproximadamente 100 espécies.

O gênero Pfaffia Mart. é neotropical com 35 espécies distribuindo-se do sul do México através dos trópicos, incluindo a Bacia Amazônica até Baía Blanca, na Argentina (Borsch 1995). Siqueira (2002) cita para a América Central e América do Sul 40 espécies, sendo 21 no Brasil. Agudelo-H. (2008) menciona 30 espécies para o neotrópico. Marchioretto (2008) confirma 20 espécies na revisão do gênero para o Brasil. O Brasil é considerado o centro de diversidade do gênero (Siqueira 1994/1995).

As espécies de Pfaffia são ervas ou subarbustos eretos ou semiprostrados, com folhas opostas ou verticiladas, inflorescências capituliformes ou em espigas, flores perfeitas e fruto cápsula monospérmica. Ocorrem em cerrados, campos rupestres, campos limpos, orlas de matas, beiras de rios e capoeiras.

Os padrões geográficos representam tipos de distribuição de organismos em uma escala que considera espaços amplos como globais e continentais, e táxons que são representados por áreas de distribuição ou centros de diversidade semelhantes. Estes padrões fitogeográficos podem ser definidos para qualquer nível taxonômico (famílias, gêneros ou espécies). Os níveis apresentam

Artigo recebido em 09/2008. Aceito para publicação em 08/2009.

${ }^{1}$ Parte da Tese de Doutorado da primeira autora.

${ }^{2}$ Programa de Pós Graduação em Botânica da Universidade Federal do Rio Grande do Sul, Av. Bento Gonçalves 9500, Bloco IV, prédio 43433, 91501-970, Porto Alegre, RS.

${ }^{3}$ Universidade Federal do Rio Grande do Sul, Depto. Botânica, Av. Bento Gonçalves, 9500, Bloco IV, prédio 43433, 91501-970, Porto Alegre, RS.

${ }^{4}$ PUC-Rio, Depto. Geografia e Meio Ambiente, R. Marquês de São Vicente 398, Gávea, 22451-041 Rio de Janeiro, RJ.

${ }^{5}$ Autor para correspondência: herbariopaca@unisinos.br; saletemarchioretto@gmail.com 
um gradiente, adequado para uma escala geográfica global, continental ou regional (Waechter 2002).

Alguns estudos fitogeográficos sobre Amaranthaceae foram realizados no Brasil. Siqueira (1994/1995) analisou e discutiu padrões de distribuição geográfica a partir dos enfoques taxonômico, areográfico e ecossistêmico. Dentro desta perspectiva incluiu o gênero Pfaffia no padrão amplo interamericano, uma vez que as espécies são encontradas somente no continente americano, sendo a maioria delas, exclusivas do Brasil. Siqueira (2004) estudou os padrões de distribuição geográfica mundiais das Amaranthaceae, fazendo comparações com os gêneros africanos e americanos, incluindo o gênero Pfaffia no padrão amplo sulamericano. Marchioretto et al. (2004) detectaram e analisaram cinco padrões de distribuição geográfica dos representantes brasileiros dos gêneros Froelichia Moench e Froelichiella R. E. Fr. Marchioretto et al. (2008a) analisaram dois padrões de distribuição geográfica dos táxons brasileiros de Hebanthe Mart. Marchioretto et al. (2008b) estudaram a biogeografia da família Amaranthaceae no Rio Grande do Sul. Os autores destacaram que o gênero Pfaffia apresenta três espécies com uma ampla distribuição nas 11 regiões fisiográficas deste estado.

O presente estudo tem por objetivo analisar de forma detalhada e comparativa os padrões de distribuição geográfica das espécies de Pfaffia ocorrentes no Brasil.

\section{Material e Métodos}

A distribuição das espécies de Pfaffia baseia-se na revisão do acervo dos seguintes herbários: ALCB, B, BHCB, BHZB, C, CEN, CEPEC, CESJ, CH, CPAP, ECA, ESA, FCAB, GUA, HAS, HB, HBR, HRB, HRCB, HTO, HUEFS, IAC, IAN, IBGE, ICN, JPB, K, MBM, MG, NY, P, PACA, R, RB, S, SJRP, SP, SPF, UB, VEN, VIC (Thiers continuamente atualizado), bem como literatura e coletas da primeira autora. Os autores das espécies mencionadas no texto encontram-se citados na Tabela 1 , abreviados segundo Brummit \& Powell (1992).

As coleções analisadas foram georreferenciadas a partir de dados das fichas de coleta ou por coordenadas geográficas obtidas no sítio eletrônico GPS Global (2007) e no programa Google Earth (4.0.2737, 31.01.2007). Mapas de distribuição geográfica foram confeccionados através do software ARC VIEW, versão 9.2 (2006). A classificação biogeográfica adotada foi a de Cabrera \& Willink (1980), que dividem a América Latina em quatro regiões, oito domínios e 24 províncias biogeográficas, destas apenas seis, inseridas domínio brasileiro (Amazônica, Atlântica, Caatinga, Cerrado, Pampeana e Paranaense).

Foi elaborada uma matriz de dados de presença e ausência das espécies para relacionar dados de riqueza e para analisar a similaridade da composição florística entre as províncias, empregando-se o índice de Jaccard, pelo método de ligação de grupos pareados, utilizando-se o programa estatístico Paleontological Statistics-PAST (Hammer et al. 2003). Os padrões de distribuição geográfica foram estabelecidos no âmbito da América do Sul.

\section{Resultados e Discussão}

As 20 espécies de Pfaffia de ocorrência confirmada para o Brasil estão apresentadas na Tabela 1. Nota-se que a maioria apresenta ocorrência exclusiva no país, de acordo com o material examinado e dados levantados na literatura (e.g. Stutzer 1935; Siqueira \& Grandi 1986; Vasconcellos 1986; Pedersen 1997; Siqueira 2002).

No entanto, o exame do vasto material de herbários e diversas coletas in situ, oportunizaram o estabelecimento de cinco padrões bem característicos para as espécies de Pfaffia. Estes padrões exibem distribuições contínuas ou não, sendo que algumas espécies apresentam uma ampla distribuição, abrangendo grande parte do território brasileiro e ainda atingindo alguns países limítrofes enquanto outras são endêmicas, sendo encontradas somente em um determinado local, de acordo com as exigências ecológicas das formações vegetacionais onde se difundem. 
Tabela 1 - Espécies de Pfaffia Mart. (Amaranthaceae) no Brasil, com sua respectiva distribuição (estados brasileiros) e hábitat.

Table 1 - Species of Pfaffia Mart. (Amaranthaceae) in Brazil, with their distribution and habitat (Brazilian states).

\begin{tabular}{lll}
\hline Espécie & Distribuição & Hábitat/ Tipo de vegetação \\
\hline Pfaffia acutifolia (Moq.) Stützer & BA, GO, MG, TO & Caatingas, cerrados, campos rupestres \\
Pfaffia aphylla Suess. & MG & Cerrados \\
Pfaffia argyrea Pedersen & MG & Campos rupestres \\
Pfaffia cipoana Marchior., Miotto \& J. C. Siqueira MG & Campos rupestres \\
Pfaffia denudata (Moq.) Kuntze & BA, DF, GO, MG, PR & Cerrados e campos rupestres \\
Pfaffia elata R. E. Fr. & GO, MS, MT & Cerrados \\
Pfaffia glabrata Mart. & GO, MG, MT, PR, SP & Cerrados \\
Pfaffia glomerata (Spreng.) Pedersen & Todo o Brasil & Beiras de rios e borda de matas \\
Pfaffia gnaphaloides (L.f.) Mart. & BA, GO, MG, MS, MT, PR, Campos limpos e campos rupestres \\
& RS, SC, SP, TO & \\
Pfaffia hirtula Mart. & MG & Campos rupestres \\
Pfaffia jubata Mart. & BA, DF, GO, MG, MT, PR, Cerrados e campos rupestres \\
& RO, SP, TO & \\
Pfaffia minarum Pedersen & GO, MG & Cerrados \\
Pfaffia rupestris Marchior., Miotto \& J. C. Siqueira MG & Campos rupestres \\
Pfaffia sarcophylla Pedersen & GO & Cerrados \\
Pfaffia sericantha (Mart.) Pedersen & BA, DF, GO, MG & Cerrados \\
Pfaffia siqueiriana Marchior. \& Miotto & BA, MG & Caatingas e campos rupestres \\
Pfaffia townsendii Pedersen & BA, GO, MG & Cerrados e campos rupestres \\
Pfaffia tuberculosa Pedersen & BA & Entre caatinga e campos rupestres \\
Pfaffia tuberosa (Spreng.) Hicken & DF, GO, MG, MS, PR, RS, Cerrados, campos rupestres e \\
& SC, SP & campos secos \\
Pfaffia velutina Mart. & MG & Cerrados e campos rupestres \\
\hline
\end{tabular}

\section{I - Padrão amplo sul-americano}

Neste padrão encontram-se cinco espécies: Pfaffia acutifolia, $P$. glabrata, $P$. glomerata, P. gnaphaloides e P. tuberosa (Fig. 1-2, Tab. 2). P. acutifolia é encontrada em três províncias biogeográficas dentro do território brasileiro, em ambientes de campos secos e úmidos nas caatingas, carrascos, campos rupestres, em altitudes entre 400-1.500 m, estendendo-se até a Bolívia (Fig. 1). P. glabrata ocorre em duas províncias, em margens de rios nos cerrados, em altitudes de aproximadamente $500 \mathrm{~m}$, podendo alcançar a Argentina e o Paraguai (Fig. 2). Com este mesmo padrão Marchioretto et al. (2004) destacaram Froelichia procera (Seub.) Pedersen ocorrendo nas mesmas províncias: Paranaense e do Cerrado. P. glomerata é encontrada nas seis províncias biogeográficas, em bordas de matas, bordas de rios e capoeiras em solos arenosos, úmidos, em altitudes que variam de 80-800 m, estendendo-se para a Argentina, Bolívia, Paraguai e Uruguai (Fig. 1). Padrão semelhante foi constatado por Marchioretto et al. (2008a) para Hebanthe eriantha (Poir.) Pedersen, embora esta espécie possua um hábito diferenciado, o hábitat se assemelha a P. glomerata, atingindo também a Argentina e Paraguai. $P$. gnaphaloides ocorre em cinco províncias, em campos limpos, secos e pedregosos, campos rupestres em altitudes que atingem de 1.000$1.500 \mathrm{~m}$. Esta espécie também apresenta a mesma abrangência da espécie anterior, não tendo sido encontrada até omomento na provínciaAmazônica (Fig. 2). P. tuberosa é encontrada em quatro províncias, em campos secos nos cerrados e campos rupestres, com solos arenosos, em altitudes que vão de 30-1.600 m atingindo a Argentina, Paraguai e Uruguai (Fig. 1). Não há registros de ocorrência desta espécie nas Províncias Amazônica e da Caatinga. Segundo 


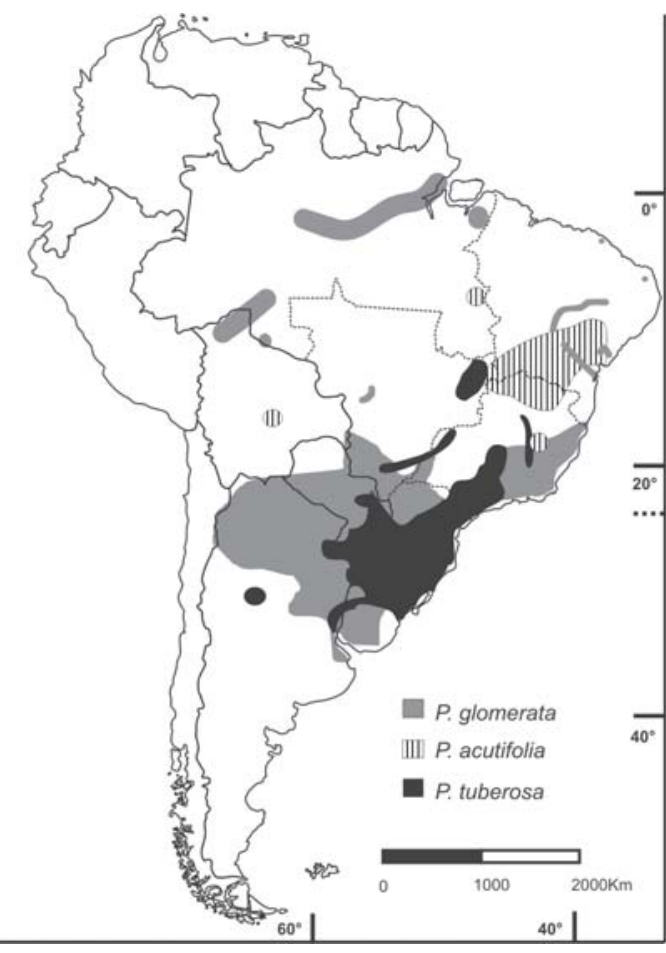

Figura 1 - Padrão amplo sul-americano, Pfaffia acutifolia (Moq.) Stützer, P. glomerata (Spreng.) Pedersen. e $P$. tuberosa (Spreng.) Hicken.

Figure 1 - Ample South-american pattern, Pfaffia acutifolia (Moq.) Stützer, P. glomerata (Spreng.) Pedersen.and P. tuberosa (Spreng.) Hicken.

Agudelo-H (2008) esta espécie foi encontrada nas regiões Amazônia, Andina e Orinoquia desde o nível do mar até elevações de 1.800 m em solos recentemente queimados.

\section{II- Padrão amplo no Brasil}

O padrão amplo é apresentado por apenas Pfaffia jubata (Fig. 3, Tab. 2). A espécie ocorre em cinco províncias, em campos secos dos cerrados e campos rupestres, em solos arenosos, com altitudes que variam entre 500 $1.700 \mathrm{~m}$, sem qualquer registro até o presente para a província Pampeana. Marchioretto et al. (2008a) destacaram este mesmo padrão para três espécies de Hebanthe, $H$. pulverulenta Mart, H. reticulata (Seub.) Borsch \& Pedersen e $H$. spicata Mart. Estas espécies mesmo não possuindo hábito e hábitat semelhantes, apresentaram o mesmo padrão, sendo que as mesmas também não foram encontradas na Província Pampeana.

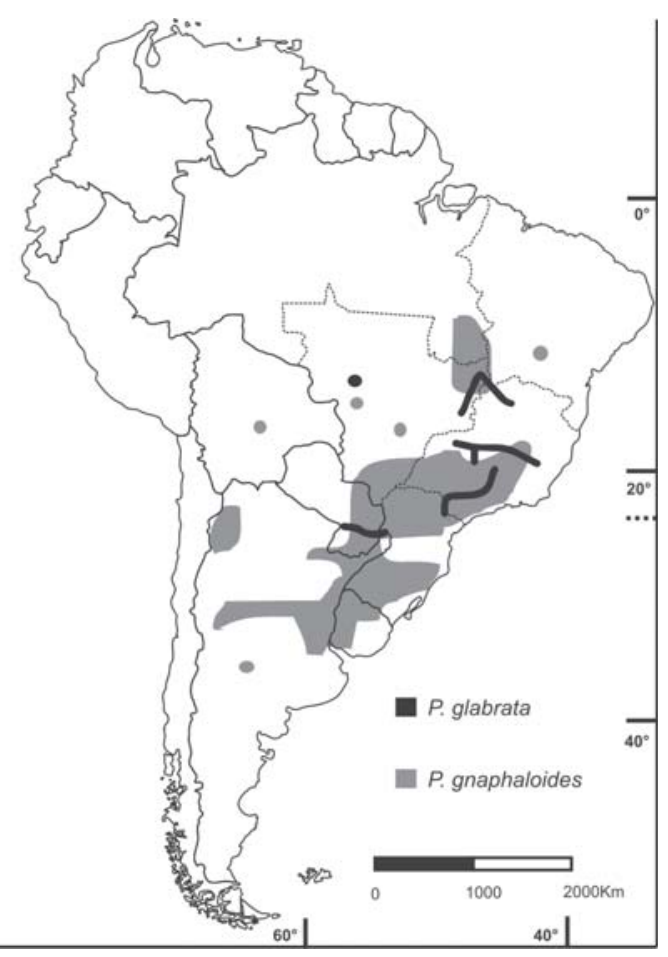

Figura 2 - Padrão amplo sul-americano, Pfaffia glabrata Mart. e P. gnaphaloides (L.f.) Mart.

Figure 2 - Ample South-american pattern Pfaffia glabrata Mart. and P. gnaphaloides (L.f.) Mart.

\section{III- Padrão moderadamente amplo no Brasil}

Três espécies podem ser incluídas neste padrão: Pfaffia denudata, $P$. townsendii e $P$. velutina (Fig. 4), sendo que todas são encontradas em duas províncias (Tab. 2). P. denudata ocorre em campos secos com solos arenosos e pedregosos nos cerrados e campos rupestres, em altitudes que atingem de $1.000-1.350 \mathrm{~m}$. $P$. townsendii é encontrada em campos secos com solos arenosos, entre rochas nos campos rupestres e cerrados, em altitudes que vão de $1.100-2.000 \mathrm{~m}$. P. velutina ocorre nos campos secos em solos arenosos, nos cerrados e campos rupestres, frequentemente encontrada após as queimadas, com altitudes que variam de 600-1.410 m.

\section{IV- Padrão restrito no Brasil}

Este padrão apresenta oito táxons, os quais são encontrados somente na Província 


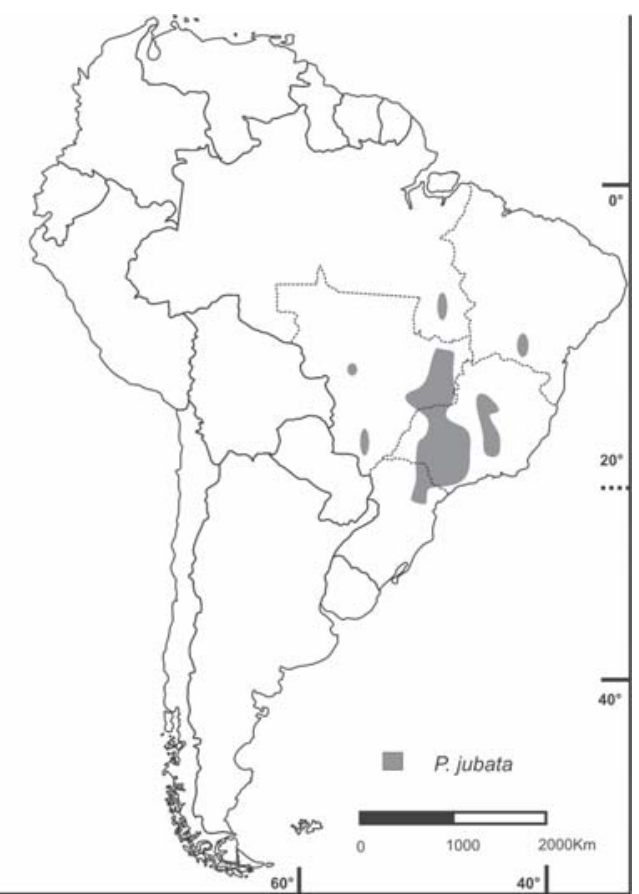

Figura 3 - Padrão amplo no Brasil, Pfaffia jubata Mart. Figure 3 - Ample Brazilian pattern, Pfaffia jubata Mart.

do Cerrado (Pfaffia aphylla, P. argyrea, P. elata, $P$. hirtula, $P$. minarum, $P$. sarcophylla, $P$. sericantha e $P$. siqueiriana) (Fig. 5-7, Tab. 2). Da mesma maneira Flores \& Tozzi (2008) evidenciaram que a Província do Cerrado apresenta um maior número de espécies nativas de Crotalaria L. (oito espécies e uma subespécie). Já Fiaschi \& Pirani apontaram 13 espécies de Schefflera J. R. \& G. Forst em formações campestres e savânicas do cerrado. Pfaffia aphylla ocorre em campos secos nos cerrados, com altitudes de aproximadamente $630 \mathrm{~m}$. Pfaffia argyrea é encontrada em campos rupestres, com solos arenosos, em altitudes que vão de 720-820 m. Pfaffia elata ocorre em campos secos nos cerrados, em altitudes que oscilam de 170-750 m. Pfaffia hirtula, encontrada em campos rupestres, em altitudes de 700-1.410 m. Pfaffia minarum ocorre nos cerrados com afloramentos rochosos, em altitudes de 600-1.200 m. Pfaffia sarcophylla localizada em cerrados, com solos serpentina, pedregosos, em altitudes de aproximadamente $965 \mathrm{~m}$. Pfaffia sericantha ocorre em cerrados ou entre cerrado e carrasco, em solos arenosos,

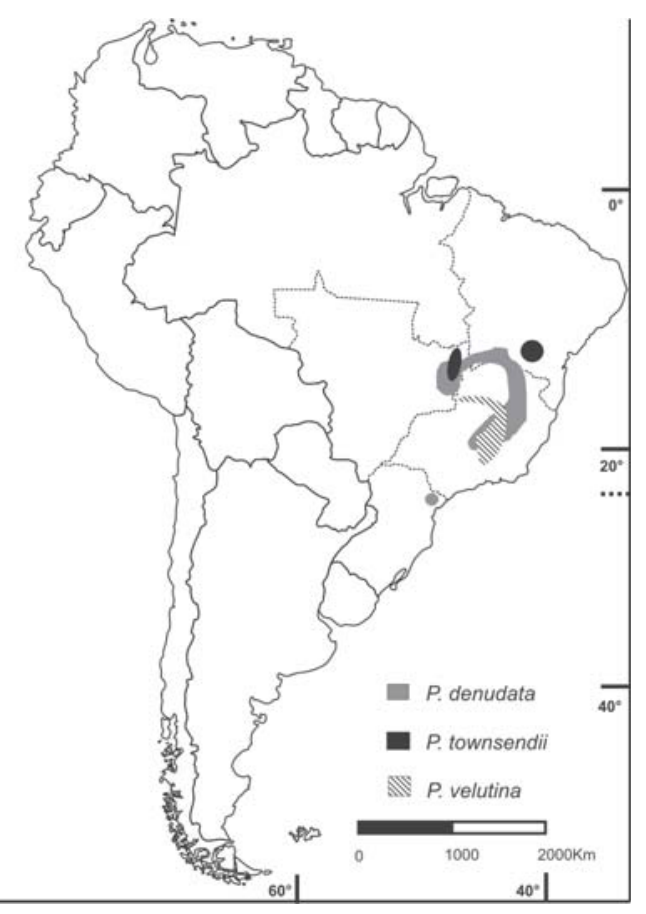

Figura 4-Padrão moderadamente amplo no Brasil; Pfaffia denudata (Moq.) Kuntze, $P$. townsendii Pedersen e $P$. velutina Mart.

Figure 4 - Moderately ample Brazilian pattern, Pfaffia denudata (Moq.) Kuntze, $P$. townsendii Pedersen and $P$. velutina Mart.

com atitudes que variam de $820-1.200 \mathrm{~m}$. ePfaffia siqueiriana é encontrada em caatingas e campos rupestres, em altitudes entre 770-832 m.

\section{V- Padrão endêmico pontual}

Encontram-se neste padrão três espécies: Pfaffia cipoana, P. rupestris e P. tuberculosa (Fig. 8, Tab. 2). P. cipoana é encontrada em Itambé do Mato Dentro, Minas Gerais, em campos rupestres, em altitudes entre 1.300$1.500 \mathrm{~m}$. Pfaffia rupestris ocorre em Rio Pardo de Minas, Minas Gerais, em campos rupestres, em altitudes de aproximadamente 750 m. Já Pfaffia tuberculosa é encontrada em Paramirim, Bahia, em ambiente de transição da caatinga com campo rupestre, com solos arenosos e pedregosos, em elevações de $640 \mathrm{~m}$. Estas três espécies apresentam um endemismo pontual, pois até o momento foram encontradas somente num único ponto da localidade citada. Alguns autores como Harley (1988, 1995), Mello-Silva (1995), Giulietti et al. (2000) e 
Tabela 2 - Matriz de presença/ausência das espécies de Pfaffia Mart. (Amaranthaceae) nas províncias biogeográficas brasileiras. 1=presente; 0 =ausente; Amazônica (AM); Atlântica (AT); Caatinga (CA); Cerrado (CE); Pampeana (PA) e Paranaense (PR).

Table 2 - Presence/absence matrix of Pfaffia Mart. (Amaranthaceae) species in the Brazilian biogeographical provinces. 1=present; 0=ausent; Amazonian (AM); Altantic (AT); Caatinga (CA); Cerrado (CE); Pampeana (PA) and Paranaese (PR).

\begin{tabular}{lccccccc}
\hline & AM & AT & CA & CE & PA & PR & Amplitude \\
\hline Pfaffia acutifolia & 1 & 0 & 1 & 1 & 0 & 0 & 3 \\
Pfaffia aphylla & 0 & 0 & 0 & 1 & 0 & 0 & 1 \\
Pfaffia argyrea & 0 & 0 & 0 & 1 & 0 & 0 & 1 \\
Pfaffia cipoana & 0 & 0 & 0 & 1 & 0 & 0 & 1 \\
Pfaffia denudata & 0 & 1 & 0 & 1 & 0 & 0 & 2 \\
Pfaffia elata & 0 & 0 & 0 & 1 & 0 & 0 & 1 \\
Pfaffia glabrata & 0 & 0 & 0 & 1 & 0 & 1 & 2 \\
Pfaffia glomerata & 1 & 1 & 1 & 1 & 1 & 1 & 6 \\
Pfaffia gnaphaloides & 0 & 1 & 1 & 1 & 1 & 1 & 5 \\
Pfaffia hirtula & 0 & 0 & 0 & 1 & 0 & 0 & 1 \\
Pfaffia jubata & 1 & 1 & 1 & 1 & 0 & 1 & 5 \\
Pfaffia minarum & 0 & 0 & 0 & 1 & 0 & 0 & 1 \\
Pfaffia rupestris & 0 & 0 & 0 & 1 & 0 & 0 & 1 \\
Pfaffia sarcophylla & 0 & 0 & 0 & 1 & 0 & 0 & 1 \\
Pfaffia sericantha & 0 & 0 & 0 & 1 & 0 & 0 & 1 \\
Pfaffia siqueiriana & 0 & 0 & 0 & 1 & 0 & 0 & 1 \\
Pfaffia townsendii & 0 & 0 & 1 & 1 & 0 & 0 & 2 \\
Pfaffia tuberculosa & 0 & 0 & 1 & 0 & 0 & 0 & 1 \\
Pfaffia tuberosa & 0 & 1 & 0 & 1 & 1 & 1 & 4 \\
Pfaffia velutina & 0 & 0 & 0 & 1 & 0 & 1 & 2 \\
Riqueza & 3 & 5 & 6 & 19 & 3 & 6 & \\
\hline
\end{tabular}

Barros (2004) discutiram a existência de padrões restritos de distribuição geográfica em espécies típicas de campos rupestres, constituindo-se casos de neondemismo ou paleoendemismo. Seguindo este mesmo entendimento, Marchioretto et al. (2004) apontaram Froelichiella grisea R. E. FR. apresentando também um padrão endêmico local dos campos rupestres da Chapada do Veadeiros, Goiás, na Província do Cerrado. Flores \& Tozzi (2008) registram espécies endêmicas de Crotolaria concentradas principalmente nos campos rupestres da Serra do Espinhaço e em formações montanhosas de Goiás. Fiaschi \& Pirani (2008) mencionaram dois casos de endemismo em Schefflera restrito a Serra da Canastra e na porção norte da Serrado espinhaço e na Serra do Cipó. Este padrão também foi reconhecido por Giulietti
\& Pirani (1988). Neste sentido Fiaschi \& Pirani (2008) afirmam que surgirão hipóteses mais consistentes a respeito da origem das espécies endêmicas de campos rupestres com a obtenção de filogenias robustas de grupos em que a diversificação foi intensa nestes locais. E com auxílio de dados geográficos precisos poderão ser sugeridos quais os principais eventos de vicariância que derivariam em padrões concordantes e qual seria a importância dos eventos de dispersão à distância para cada grupo considerado. A idade dos clados nessas filogenias, baseados em datação molecular e/ou com raros testemunhos fósseis disponíveis poderá avançar com mais garantia nessa consideração.

De acordo com estes padrões constatouse um predomínio de espécies de Pfaffia nos cerrados e campos rupestres, sendo que Minas 


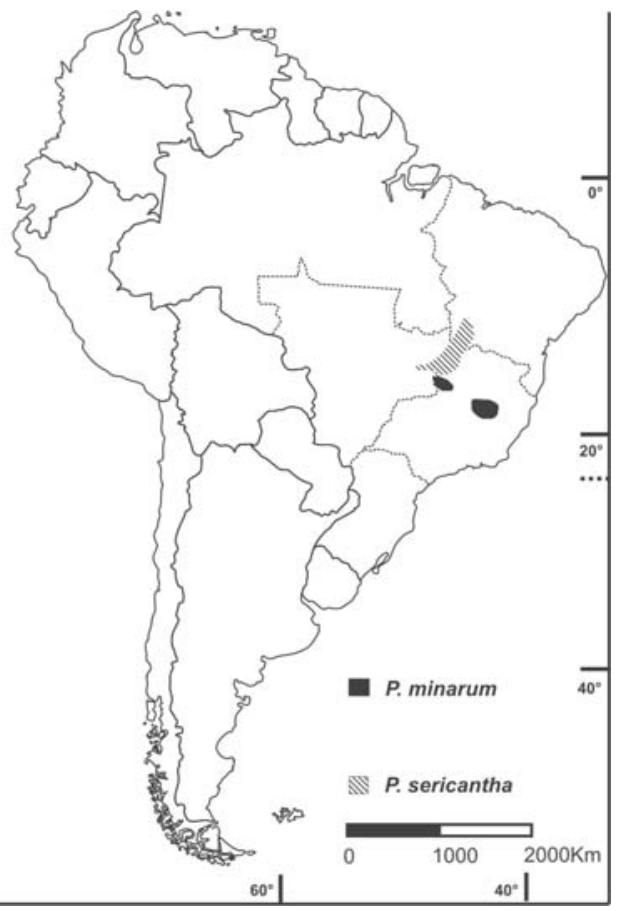

Figura 5 - Padrão restrito no Brasil, Pfaffia minarum Pedersen, P. sericantha (Mart.) Pedersen.

Figure 5 - Restrict Brazilian Pattern, Pfaffia minarum Pedersen, P. sericantha (Mart.) Pedersen.

Gerais foi o estado que apresentou o maior número de táxons (Tab. 1). Segundo a classificação adotada por Cabrera \& Willink (1980), tais espécies estariam localizadas na província do Cerrado. Embora as várias classificações como tipos vegetacionais (Veloso et al. 1991), regiões fitoecológicas ou áreas (IBGE 2004a), biomas (IBGE 2004b; WWF 2005) e províncias (Cabrera \& Willink 1980), não destaquem os campos rupestres como um bioma, subentende-se que os mesmos estejam incluídos na região, bioma ou província do Cerrado. Para o bioma Cerrado são descritos 11 tipos principais de vegetação, enquadrados em: formações florestais (mata ciliar, mata de galeria, mata seca e cerradão), savânicas (cerrado stricto sensu, parque de cerrado, palmeiral e vereda) e campestres (campo sujo, campo limpo e campo rupestre) (Ribeiro \& Walter 1998).

Segundo Coutinho (2006), modernamente, o cerrado é considerado como sendo uma savana e que este termo possui dois conceitos:

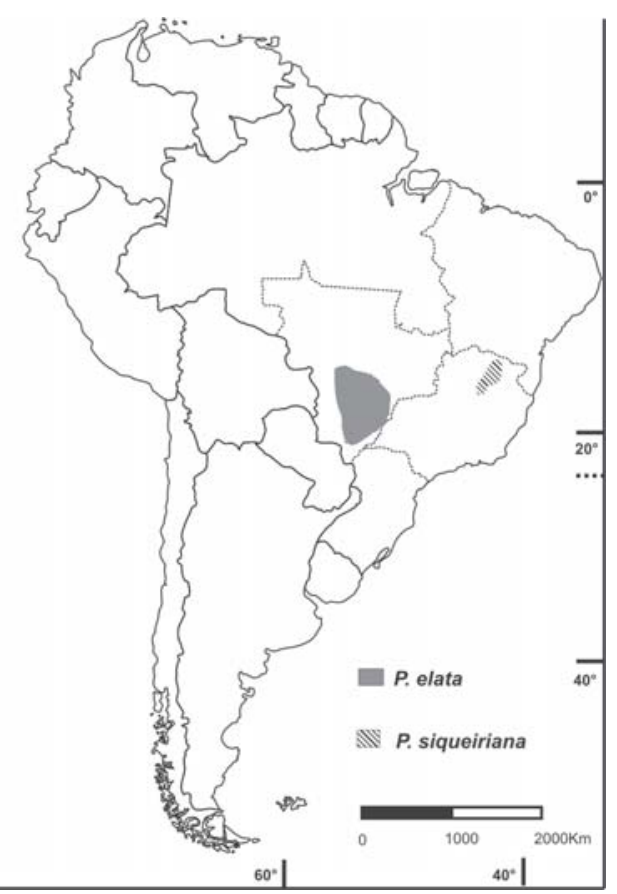

Figura 6 - Padrão restrito no Brasil; Pfaffia elata R. E. Fr. e P. siqueiriana Marchioretto \& Miotto.

Figure 6 - Restrict Brazilian Pattern, Pfaffia elata R. E. Fr. and P. siqueiriana Marchioretto \& Miotto.

um de natureza fitofisionômica e outro referente a um grande ecossistema, com seu tipo particular de vegetação. Coutinho (l.c.) comenta que o conceito fiel de cerrado adotado por diversos autores é o de que cerrado não é um bioma único, mas um complexo de biomas, formado por um mosaico de comunidades pertencentes a um gradiente de formações ecologicamente relacionadas que abrangem desde o campo limpo até o cerradão. A origem do cerrado é discutida por vários autores, entre eles, Hueck (1957), Warming (1973), Coutinho (1992), Ribeiro \& Walter (1998), sendo que uma das teorias é a de que a vegetação do cerrado teria uma possível origem antrópica, devido ao uso frequente do fogo. No entanto, a homogeneidade encontrada no mesmo, com elementos comuns que se estendem da Amazônia até Paraná e a ilha encontrada na floresta amazônica induz tratar-se de uma formação primitiva.

Segundo Brandão (2000), o aspecto visual do cerrado é muito característico e divergente das demais formações, que se pode supor que 


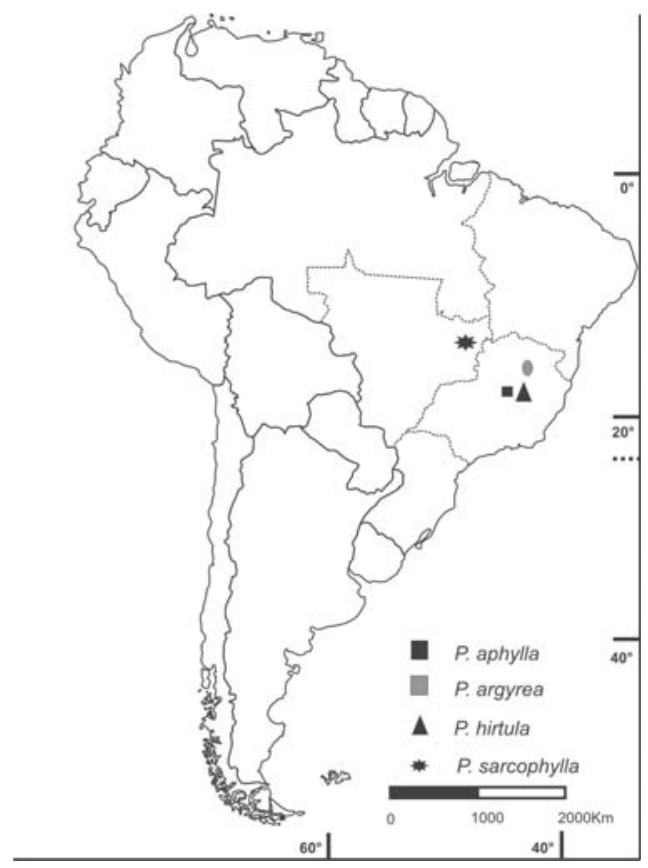

Figura 7 - Padrão restrito no Brasil, Pfaffia aphylla Suess., $P$. argyrea Pedersen, $P$. hirtula Mart.,e $P$. sarcophylla Pedersen.

Figure 7 - Restrict Brazilian pattern, Pfaffia aphylla Suess., $P$. argyrea Pedersen, P. hirtula Mart. and P. sarcophylla Pedersen.

seja resultado de uma adaptação muito antiga às condições ecológicas, isto é, o cerrado seria remanescente de um revestimento florístico muito antigo, desta maneira explicando as ilhas encontradas além dos limites do bioma. Estudos recentes têm revelado que a flora do cerrado apresenta um elevado índice de diversidade de angiospermas (Castro et al. 1999). Sendo que alguns autores como Ratter et al. (1996), Oliveira-Filho \& Ratter (2002) afirmaram haver uma heterogenidade florística, servindo esta de base para o reconhecimento de agrupamentos florísticos neste domínio. De acordo com Cabrera \& Willink (1980) a província biogeográfica do Cerrado abrange grande parte do território brasileiro, apresentando um estrato arbóreo-arbustivo importante e outro herbáceo muito rico em leguminosas, gramíneas e numerosas amarantáceas.

Tratando-se dos campos rupestres, estes se localizam principalmente na cadeia do Espinhaço, em Minas Gerais e na Bahia, e em algumas disjunções nas serras de Goiás. Em
Marchioretto, M. S., Miotto, S. T. S. \& Siqueira, J. C.

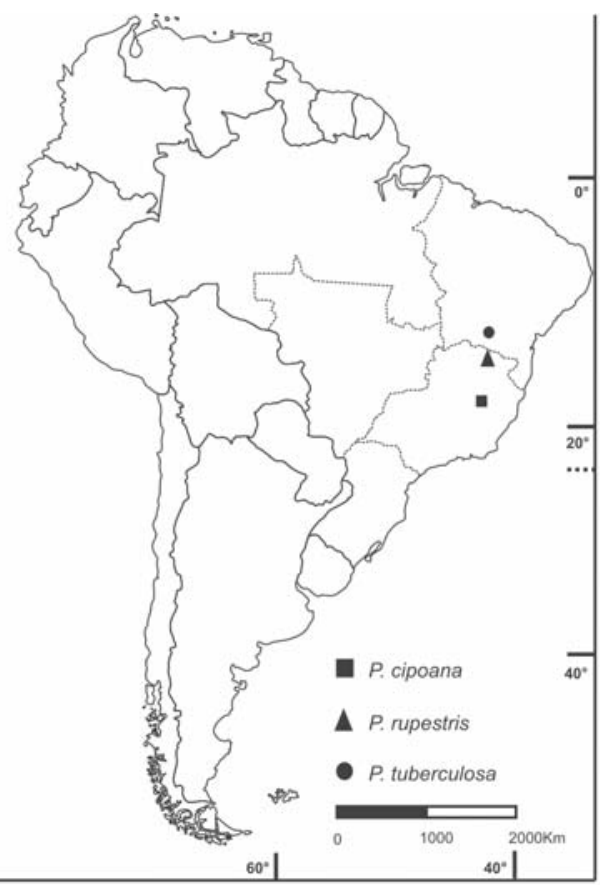

Figura 8 - Padrão endêmico pontual, Pfaffia cipoana Marchioretto, Miotto \& Siqueira, P. rupestris Marchioretto, Mioto \& Siqueira e P. tuberculosa Pedersen.

Figure 8 - Endemic punctual pattern, Pfaffia cipoana Marchioretto, Miotto \& Siqueira, P. rupestris Marchioretto, Mioto \& Siqueira and P. tuberculosa Pedersen.

Minas Gerais, estes estão cercados pelo cerrado, enquanto que na Bahia, o seu relacionamento principal é com a caatinga (Giulietti et al. 1997). A flora dos campos rupestres da Cadeia do Espinhaço, em Minas Gerais, é formada principalmente por gêneros e espécies endêmicos, com áreas de distribuição muito restrita. Esta flora mostra conexões com outras áreas da América do Sul, como por exemplo, as serras do Planalto Central e as dos "tepuis" da Venezuela, podendo ter espécies em comum com a flora do cerrado (Menezes \& Giulietti 2000). A distribuição geográfica descontínua em serras da Cadeia do Espinhaço é recorrente em plantas arbustivas e herbáceas dos campos rupestres (Giulietti \& Pirani 1988), corroborado pelas espécies de Pfaffia.

Menezes \& Giulietti (2000) referem que algumas famílias, gêneros ou espécies ocorrem frequentemente sobre solos arenosos ou dos substratos rochosos, que formam grande parte 
dos campos rupestres, ocorrendo grandes e vistosas populações, contribuindo para a fisionomia geral da vegetação, dentre elas destacam-se as Asteraceae, Bromeliaceae, Cyperaceae, Eriocaulaceae, Lamiaceae, Leguminosae, Lythraceae, Melastomataceae, Myrtaceae, Orchidaceae, Poaceae, Rubiaceae e Velloziaceae. As citadas autoras não incluíram nesta listagem as Amaranthaceae que também apresentam esta peculiaridade, principalmente, as espécies de Pfaffia que ocorrem, basicamente, nos cerrados e campos rupestres. Sabe-se que suas plantas possuem características adaptativas a estes biomas como os sistemas subterrâneos, a dispersão anemocórica e o pirofitismo (Siqueira 2007).

As espécies de Pfaffia, mais raras ou endêmicas são quase que exclusivas dos campos rupestres. Estes mesmo localizados principalmente em áreas mais distantes das ações antrópicas, geralmente preservados em parques, reservas ou unidades de conservação, também vêm sofrendo a redução de suas populações, devido às queimadas e ao pastoreio. Isto pode ser verificado ao analisar-se os padrões de distribuição e as áreas de ocorrência de Pfaffia argyrea, $P$. hirtula, $P$. siqueiriana, $P$. cipoana e $P$. rupestris. Este fato é contestado por Ribeiro \& Walter (1998), argumentando que a composição florística em campos rupestres pode variar em poucos metros de distância e a densidade das espécies depende da profundidade do solo, fertilidade e disponibilidade de água, predominando a vegetação herbácea. Outras espécies como Pfaffia acutifolia, P. denudata, $P$. jubata, $P$. townsendii e $P$. velutina, que ocorrem tanto em cerrados como em campos rupestres, embora apresentem uma distribuição mais ampla no Brasil, tornaram-se cada vez menos frequentes em função da forte ação antrópica e ou fogo, estando sujeitas a desaparecerem com a progressiva destruição do bioma Cerrado (Siqueira 2007).

A análise da distribuição geográfica das espécies de Pfaffia nas províncias biogeográficas (Tab. 2), permite observar um gradiente de riqueza onde a Província do Cerrado, a mais rica (19 espécies) (Fig. 9), sendo as mais pobres

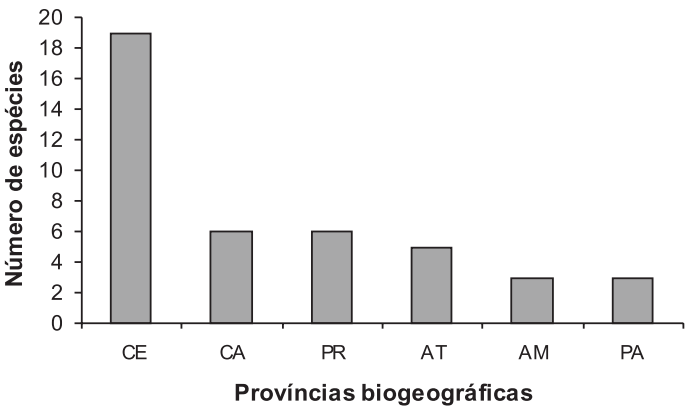

Figura 9 - Riqueza de espécies de Pfaffia Mart. (Amaranthacae) nas províncias biogeográficas brasileiras. Cerrado (CE), Caatinga (CA), Paranaense (PR), Atlântica (AT), Amazônica (AM) e Pampeana (PA).

Figure 9 - Species richness of Pfaffia Mart. (Amaranthacae) in the Brazilian biogeographical provinces. Cerrado (CE), Caatinga (CA), Paranaense (PR), Atlantic (AT), Amazonian (AM) and Pampaeana (PA).

as Províncias Amazônica e Pampeana (três espécies cada). Pfaffia tuberculosa é restrita à Província da Caatinga e Pfaffia aphylla, $P$. argyrea, $P$. cipoana, P. elata, $P$. hirtula, $P$. minarum, $P$. rupestris, $P$. sarcophylla e $P$. siqueiriana restritas à Província do Cerrado. Pfaffia jubata apresenta ampla distribuição, ocorrendo nas Províncias Atlântica, Amazônica, Caatinga, Cerrado e Paranaense. Pfaffia gnaphaloides mostra distribuição semelhante, não ocorrendo na Província Amazônica, atingindo, porém, a Província Pampeana. Somente $P$. glomerata ocorre nas seis províncias biogeográficas.

Muitas destas espécies são exclusivas dos cerrados e campos rupestres, principalmente de Minas Gerais, sendo que o cerrado pode ser considerado o centro de diversidade e de endemismo do gênero. $O$ centro de endemismo da Região Sudeste, principalmente no estado de Minas Gerais, sugere que o gênero teve capacidade para se adaptar às condições climáticas e edáficas dos cerrados e campos rupestres desta região. O contrário pode ocorrer com um baixo nível de riqueza de espécies de outras famílias como é o caso de Gesneriaceae que, segundo Chautems (2003), é extremamente baixa no cerrado devido à dificuldade de adaptação às condições climáticas nesta região. Neste sentido Spellerberger \& 
Sawyer (2000) afirmam que a distribuição geográfica de uma espécie está diretamente associada a fatores ecológicos e históricos. Embora cada espécie tenha sua própria distribuição, padrões repetitivos são comuns, sendo que alguns refletem conexões e paleoclimas, enquanto outros indicam limites impostos por condicionantes ambientais atuais (Brown \& Gibson 1983). A grande maioria das espécies tropicais não tem sua distribuição geográfica conhecida e, em geral, para poucas destas espécies têm-se informações disponíveis em nível de Brasil (Siqueira 2005). As informações contidas nas coleções, muitas vezes não refletem a verdadeira distribuição do grupo, pois geralmente, suas coletas são restritas a determinados locais de fácil acesso, ou ao longo de caminhos já existentes, na extensão de cursos de água, em locais de fácil hospedagem, independentes de meios de locomoção em determinados meses do ano, mostrando desta maneira "falsas lacunas" (Ferrier 2002). Associado a estes fatores temse a deficiência da base amostral dos herbários nacionais em relação a extensas áreas territoriais.

Segundo Myers et al. (2000) o número de angiospermas no cerrado pode chegar a 10.000 , sendo que o nível de endemismo pode alcançar $44 \%$ para as plantas vasculares. Expressiva parte dessa riqueza pode ser explicada pela existência de um mosaico natural de ecossistemas que compartilham a paisagem do cerrado (Aguiar et al. 2004). Para Mendonça et al. (1998), 35\% das plantas do cerrado são de formação de cerrado sensu stricto, 30\% das espécies são de mata de galeria, $25 \%$ de áreas campestres e 10\% ainda não estão classificadas. Cabe ressaltar que os registros para plantas no bioma Cerrado, são bastante deficientes e concentrados em poucas localidades, como São Paulo (Shepherd 2000) e Brasília (Mendonça et al. 1998).

A exploração dos cerrados data de séculos passados, sendo que em décadas recentes a expansão agrícola iniciou uma acelerada ocupação do referido bioma, baseada na exploração extrativista e predatória. A explosão agrícola sobre o cerrado encontrou uma região de solos, caracteristicamenteácidos e com baixo teor nutricional. Estes, na maioria dos casos, não são submetidos a qualquer trato cultural, além de serem expostos a ciclos periódicos de queimadas. O desmatamento, para a retirada de madeira e produção de carvão vegetal, foi e ainda é uma atividade que antecedeu e viabilizou a ocupação agropecuária do cerrado e concomitantemente o aumento das atividades agropastoris. $\mathrm{O}$ acelerado ritmo do processo de urbanização na região, também tem contribuído para o aumento da pressão sobre as áreas ainda não ocupadas do Cerrado.

A vegetação herbácea e subarbustiva dos cerrados é formada por espécies predominantemente perenes, com órgãos subterrâneos de resistência, como bulbos, xilopódios, sóboles, etc., que lhes garantem sobreviver à seca e ao fogo. Suas raízes são geralmente superficiais, atingindo cerca de $30 \mathrm{~cm}$. Os ramos aéreos são anuais, secando e morrendo durante a estação seca. Formam-se algumas toneladas de biomassa por ano, facilmente inflamável, favorecendo assim a ocorrência e a propagação das queimadas nos cerrados. Este fato é facilmente constatado na família Amaranthaceae, principalmente em algumas espécies dos gêneros Alternanthera Forssk., Gomphrena L. e Pfaffia, cujos sistemas subterrâneos são eliminados com a destruição sistemática dos cerrados e a retirada do estrato herbáceo-arbustivo, impedindo as reproduções sexuada e vegetativa, esta última ocorrendo após as queimadas através da rebrota dos sistemas subterrâneos (Siqueira 2007).

Desta maneira, é cada vez menos frequente a localização de espécies, principalmente, de Pfaffia, que apresentam padrões de distribuição mais restritos ou endêmicos aos cerrados (Siqueira 2007). César (1980) ao estudar os efeitos causados pela queima e corte da vegetação de campo sujo no cerrado stricto sensu, na Região Centro-Oeste (Brasília), observou 46 espécies, sendo que destas, apenas seis floresciam exclusivamente depois do fogo, entre elas destacou Pfaffia jubata.

Como ocorre com alguns táxons tropicais, a riqueza de espécies de Pfaffia decresce no sentido norte-sul do Brasil, à medida que estas 
atingem as províncias com predomínio maior da floresta subtropical e pluvial e dos campos sulinos, sendo que geralmente os táxons encontrados nestas províncias são os que apresentam uma maior amplitude geográfica como é o caso de Pfaffia glomerata, $P$. gnaphaloides e $P$. tuberosa. Esta tendência também foi verificada primeiramente por Rambo (1960) o qual argumentou que, no Rio Grande do Sul, o número de espécies de famílias tipicamente tropicais diminui. Waechter (1998) evidenciou a mesma tendência para orquídeas epifíticas e Ritter \& Weachter (2004) também observaram em Mikania Willd. (Asteraceae).

A análise de agrupamento (Fig. 10) evidenciou a formação de dois grandes grupos: um primeiro, formado pelo maior número de espécies (19), abrangendo a Província do Cerrado e um segundo, formado por dois subgrupos, sendo um constituído pelas espécies mais setentrionais que ocorrem nas Províncias da Caatinga e Amazônica e o outro subgrupo representado principalmente por espécies mais meridionais presentes nas províncias Pampeana, Atlântica e Paranaense.

O resultado mais importante na análise florística, evidenciado no dendrograma (Fig. 10), foi a separação das espécies que ocorrem na Província do Cerrado, das que ocorrem nas outras províncias. Fato este também verificado por Cabrera \& Willink (1980), observando que a província biogeográfica do Cerrado abrange grande parte do território brasileiro, apresentando um estrato arbustivo importante e outro herbáceo muito rico em leguminosas, gramíneas e numerosas amarantáceas.

Verificou-se uma similaridade maior entre as províncias da Caatinga e Amazônica, por apresentarem espécies com ampla distribuição, adaptando-se muito bem em ambientes mais úmidos, como é o caso de Pfaffia glomerata, que ocorre de norte ao sul do Brasil em bordas de rios e beira de matas, sendo, também cultivada em vários estados brasileiros para venda de suas raízes, com uso medicinal. As espécies que ocorrem nestas províncias em ambientes mais secos foram $P$. acutifolia e $P$.

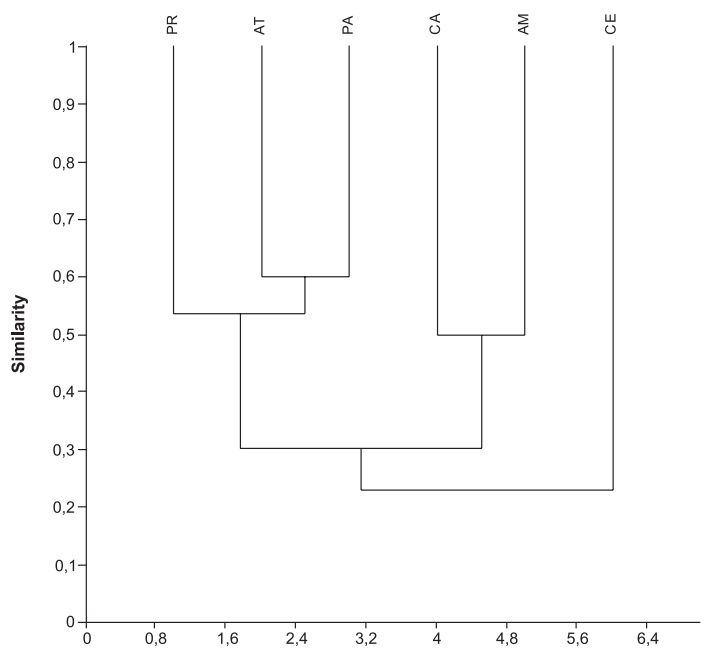

Figura 10 - Dendrograma de similaridade florística das espécies de Pfaffia Mart. (Amaranthaceae) nas diferentes províncias biogeográficas do Brasil. Amazônica (AM), Atlântica (AT), Caatinga (CA), Cerrado (CE), Pampeana (PA) e Paranaense (PR).

Figure 10 - Dendrogram of floristic similarity of the species of Pfaffia Mart. (Amaranthaceae) in different Brazilian biogeographical provinces. Amazonian (AM), Atlantic (AT), Caatinga(CA), Cerrado (CE), Pampeana (PA) e Paranaense (PR).

jubata. Esta constatação pode ser justificada uma vez que segundo o WWF (2005), existe uma transição entre os biomas Amazônia e Caatinga com a ocorrência de elementos comuns aos dois biomas.

A maior similaridade na composição florística constatada entre as províncias Paranaense, Atlântica e Pampeana confirma a continuidade de seus limites, além da ocorrência das espécies que apresentaram uma distribuição bastante ampla no Brasil como é o caso de P. glomerata, P. gnaphaloides e P. tuberosa.

As espécies de Pfaffia são encontradas em cerrados e em campos rupestres, áreas consideradas mais críticas e ameaçadas devidas sua diversidade e endemismos. Aárea mais rica em endemismo para este grupo foi a Cadeia do Espinhaço em Minas Gerais, sugere a capacidade das espécies de Pfaffia adaptarem-se aos fatores climáticos e edáficos dos cerrados e campos rupestres. Estudos filogenéticos entre as espécies poderão contribuir para um melhor conhecimento e 
entendimento dos eventos responsáveis pelos padrões atualmente observados e mapeados. Como o bioma Cerrado é considerado um dos “hotspots” para conservação da biodiversidade mundial, reforça a necessidade de estratégias de conservação nestes ecosssitemas potencialmente ameaçados.

\section{Agradecimentos}

Ao Dr. Pedro Ignácio Schmitz, Diretor do Instituto Anchietano de Pesquisas pelo apoio, incentivo e infra-estrutura. Aos Dr. Albano Backes e Dr. Paulo Gunter Windisch pelas importantes sugestões. Aos colegas Fabiana de Azevedo e Fúlvio Vinícius Arnt pelas contribuições na plotagem e confecção dos mapas.

\section{REFERÊNCIAS BIBLIOGRÁFICAS}

Agudelo-H, C. A. 2008. Amaranthaceae. Flora de Colombia. $n^{\circ} 23$. Instituto de Ciencias Naturales, Universidad Nacional de Colombia, Bogotá. 138p.

Aguiar, L. M. S.; Machado, R. B. \& MarinhoFilho, J. 2004. A diversidade biológica do cerrado. In: Aguiar, L. M. S. \& Camargo A. J.A. (org.). Cerrado: ecologia e caracterização. Embrapa Informação Tecnológica, Brasília. Pp 17-39.

APG II. The Angiosperm Phylogeny Group 2003. An update of Angiosperm Phylogeny Group classification for the orders and families of flowering plants: APG II. Botanical Journal of the Linnean Society 141: 399-436.

Barros, F. 2004. Distribuição geográfica de orquídeas do Planalto Central do Brasil. In: Barros, F. \& Kerbauy, G. B. (eds.). Orquidologia sul-americana: uma compilação científica. Secretaria do Meio Ambiente, São Paulo. Pp. 147-153.

Borsch, T. 1995. Three New Combinations in Pfaffia (Amaranthaceae) from the New World Tropics. Novon 5: 230-233.

Brandão, M. 2000. Cerrado. In: Mendonça, M. P. \& Lins, L. V (org.). Lista vermelha das espécies ameaçadas de extinção da flora de Minas Gerais. Fundação Biodiversitas e Fundação Zôo-Botânica de Belo Horizonte, Belo Horizonte. Pp. 55-63

Brown, J. H. \& Gibson, A. C. 1983. Distribution patterns of plants. Biogeography. C.V. Mostely, St. Louis. 643p.

Brummit, R. K. \& Powell, C. E. 1992. Authors of plant names. Royal Botanic Gardens, Kew. 732p.

Cabrera, A. L. \& Willink, A. 1980. Biogeografia de America Latina. 2 ed. OEA, Washington. 122p.

Castro, A. A. J. F.; Martins, F. R.; Tamashiro, J. Y. \& Shephered, G. J. 1999. How rich is the flora of Brazilian cerrados? Annals of the Missouri Botanical Garden 86:192-224.

César, H. L. 1980. Efeitos da queima e corte sobre a vegetação de um campo sujo na Fazenda Água Limpa, Brasília-DF. Dissertação de Mestrado. Universidade de Brasília, Brasília. 59p.

Chautems, A. 2003. Gesneriaceae. In: Cavalcanti, T. B. \& Ramos, A. E. (orgs.). Flora do Distrito Federal, Brasil. v. 3. Embrapa Recursos Genéticos e Biotecnologia, Brasília. 240p.

Coutinho, L. M. 1992. O cerrado e a ecologia do fogo. Ciência Hoje (Volume especial Eco-Brasil). Pp.130-138.

Coutinho, L. M. 2006. O conceito de bioma. Acta Botanica Brasilica 20(1): 13-23.

Ferrier, S. 2002. Mapping spatial pattern in biodiversity for regional conservation plannning: where to from here? Systematic Biology 51(2): 331-363.

Fiaschi, P. \& Pirani, J. R. 2008. Padrões de distribuição geográfica das espécies de Schefflera J. R. Forst. \& G. Forst (Araliaceae) do Brasil extra-amazônico. Revista Brasileira de Botânica 31(4): 633-644.

Flores, A. S. \& Tozzi, A. M. G. A. 2008. Phytogeographical patterns of Crotolaria species (Leguminosae-Papilionoidae) in Brasil. Rodriguésia 59(3): 477-486.

Giullietti, A. M. \& Pirani, J. R. 1988. Patterns of geographic distribution of some plant species from the Espinhaço Range, Minas Gerais and Bahia, Brazil. In: Vanzolini, P. 
E. \& Heyer, W. R. (eds). Proceedings of a workshop on Neotropical distribution patterns. Academia Brasileira de Ciências, Rio de Janeiro. Pp. 39-69.

Giullietti, A. M.; Pirani, J. R. \& Harley, R. M. 1997. Espinhaço range region - Eastern Brazil. In: Davis, S. D. et al. (eds.). Centres of plant diversity, v. 2. IUCN, Cambridge. Pp. 397-404.

Giullietti, A. M.; Harley, R. M.; Queiroz, L. P.; Wanderley, M. G. L. \& Pirani, J. R. 2000. Caracterização e endemismos nos campos rupestres da Cadeia do Espinhaço. In: Cavalcanti, T. B. \& Walter, B. M. T. (orgs.). Tópicos atuais em botânica: palestras convidadas do $51^{\circ}$ Congresso Nacional de Botânica Embrapa/SBB, Brasília. Pp.311-318.

GPS Global. http://www.gpsglobal.com.br/. Acesso em: 07.08.2007.

Hammer, O.; Harper, D. A. T. \& Ryan, P. D. 2003. Paleontological Statistics- PAST. Version 1.18. http://folk.uio.no/ohammer/ past.

Harley, R. M. 1988. Evolution and distribution of Eriope (Labiatae) and its relatives in Brazil. In: Vanzolini, P. E. \& Heyer, W. R. (eds.). Proceedings of a workshop on Neotropical distribution patterns Academia Brasileira de Ciências, Rio de Janeiro. Pp. 71-120.

Harley, R. M. 1995. Introdução. In: Stannard, B. L. (ed.). Flora of the Pico das AlmasChapada Diamantina, Bahia, Brazil. Royal Botanic Garden, Kew. Pp. 43-78.

Hueck, K. 1957. Sobre a origem dos campos cerrados no Brasil e algumas novas observações no seu limitemeridional. Revista Brasileira de Geografia 19(1): 67-82.

IBGE. 2004a. ftp://geoftp.ibge.gov.br/mapas/ tematicos/mapas_murais/vegetacao.pdf. (Acesso em 03/2008).

IBGE. 2004b. ftp://geoftp.ibge.gov.br/mapas/ tematicos/mapas_murais/biomas.pdf. (Acesso em 03/2008).

Judd, W. S.; Campbell, C. S.; Kellogg, E. A.; Stevens, P. F. \& Donoghue, M. J. 2002. Plant systematics. A phylogenetic approach. 2 ed. Sunderland, Sinauer Associates. 576p.
Marchioretto, M. S. 2008. Os gêneros Hebanthe Mart. e Pfaffia Mart. (Amaranthaceae) no Brasil. Tese de Doutorado. Universidade Federal do Rio Grande do Sul, Porto Alegre. 255p.

Marchioretto, M. S.; Windisch, P. G. \& Siqueira, J. C. 2004. Padrões de distribuição geográfica das espécies de Froelichia Moench e Froelichiella R.E. Fries (Amaranthaceae) no Brasil. Iheringia Sér. Bot. 59(2): 149-159.

Marchioretto, M. S.; Miotto, S. T. S. \& Siqueira, J. C. 2008 a. Padrões de distribuição geográfica dos táxons brasileiros de Hebanthe Mart. (Amaranthaceae). Pesquisas-Botânica 59: 159-170.

Marchioretto, M. S; Azevedo, F.; Josende, M. V. F \& Schnorr, D. M. 2008 b. Biogeografia da família Amaranthaceae no Rio Grande do Sul. Pesquisas-Botânica 59: 171-190.

Mello-Silva, R. 1995. Aspectos taxonômicos, biogeográficos, morfológicos e biológicos das Velloziaceae de Grão Mogol, Minas Gerais, Brasil. Boletim de Botânica da Universidade de São Paulo 14: 49-79.

Mendonça, R. C.; Felfili, J. M.; Walter, B. M. T.; Silva-Junior, M. C.; Rezende, A. V.; Filgueira, T. S. \& Nogueira, P. E. 1998. Flora Vascular do Cerrado. In: Sano, S. M. \& Almeida, S. P. (ed.). Cerrado: ambiente e flora. Embrapa Cerrados, Planaltina. Pp. 289-556.

Menezes, N. L. \& Giulietti, A. M. 2000. Campos Rupestres. In: Mendonça, M. P. \& Lins, L. V. (org.). Lista vermelha das espécies ameaçadas de extinção da flora de Minas Gerais. Belo Horizonte, Fundação Biodiversitas e Fundação Zôo-Botânica de Belo Horizonte. Pp. 65-73.

Myers, N.; Mittermeier, R. A.; Mittermeier, C. G.; Fonseca, G. A. B. \& Kents, J. 2000. Biodiversity hotspots for conservation priorities. Nature 403: 853-858.

Oliveira-Filho, A. T. \& Ratter, J. A. 2002. Vegetation physiognomies and woody flora of the Cerrrado Biome. In: Oliveira, P. S. \& Marquis, R. J. (eds.). The Cerrados 
of Brazil: ecology and natural history of a Neotropical savanna Columbia University Press, New York. Pp. 91-120.

Pedersen, T. M. 1997. Studies in South American Amaranthaceae. I. Bul. Mus. Natl. Hist. Nat. Sér.3. Adansonia 19 (2): 217-251.

Rambo, B. 1960. Die sudgrenze des brasilianischen Regenwaldes. Pesquisas 8: 5-41.

Rather, J. A.; Bridgewatter, S.; Atkinson, R. \& Ribeiro, J. F. 1996. Analysis of the floristic composition of the Brazilian Cerrado vegetation II: comparison of woody vegetation of 98 areas. Edinburg Journal of Botany 53:153-180.

Ribeiro, J. F. \& Walter, B. M. T. 1998. Fitofisionomias do bioma Cerrado. In: Sano, S. M.; Almeida, S. P. (ed.). Cerrado: ambiente e flora. Embrapa Cerrados, Planaltina. Pp. 89-152.

Ritter, M. R. \& Waechter, J. L. 2004. Biogeografia do gênero Mikania Willd. (Asteraceae) no Rio Grande do Sul, Brasil. Acta Botanica Brasilica 18(3): 643-652.

Shepherd, G. J. 2000. Conhecimento e diversidade de plantas terrestres do Brasil. Brasília. 53p.

Siqueira, J. C. 1994/1995. Fitogeografia das Amaranthaceae Brasileiras. PesquisasBotânica 45:5-21.

Siqueira, J. C. 2002. Amaranthaceae. In: Wanderley, M. G. L.; Shepherd, G. \& Giulietti, A.M. (orgs). Flora fanerogâmica do estado de São Paulo. FAPESPHUCITEC, São Paulo. Pp. 11-30.

Siqueira, J. C. 2004. Amaranthaceae: padrões de distribuição geográfica e aspectos comparativos dos gêneros africanos e sul-americanos. Pesquisas-Botânica 55: 177-185.

Siqueira, J. C. 2007. O bioma cerrado e a preservação de grupos taxonômicos: um olhar sobre as Amaranthaceae. PesquisasBotânica 58: 389-394.

Siqueira, J. C. \& Grandi, T. S. M. 1986. O gênero Pfaffia Mart. (Amaranthaceae) nos cerrados e campos rupestres de Minas Gerais. Acta Biologica Leopoldensia 8(2): 213-230.
Siqueira, M. F. 2005. Uso da modelagem de nicho fundamental na avaliação do padrão de distribuição geográfica de espécies vegetais. Tese de Doutorado. Universidade de São Paulo, São Carlos. 106p.

Souza, V. C. \& Lorenzi, H. 2008. Botânica sistemática: guia ilustrado para identificação das famílias de Angiospermas da flora brasileira, baseado em APG II. Instituto Plantarum, Nova Odessa. 704p.

Spellerberger, L. F. \& Sawyer, J. W. D. 2000. An introduction to applied biogeography. University Press, Cambridge. 243p.

Stutzer, O. 1935. Die Gattung Pfaffia mit einem Anhag neur Arten von Alternanthera. Feddes Repertorium Specierum Novarum Regni Vegetabilis 88: 1-49.

Thiers, B. [continuously updated]. Index Herbariorum: A global directory of public herbaria and associated staff. New York Botanical Garden's Virtual Herbarium. http://sweetgum.nybg.org/ih/. Acesso em 08/2008.

Vasconcellos, J. M. O. 1986. Amaranthaceae do Rio Grande do Sul, Brasil. -V. Gêneros Pfaffia Mart. e Gomphrena Mart. Roessléria 8 (2): 75-127.

Veloso, H. P.; Rangel Filho, A. L. R \& Lima, J. C. A. 1991. Classificação da vegetação brasileira, adaptada a um sistema universal. Instituto Brasileiro de Geografia e Estatística, Rio de Janeiro. 123p.

Waechter, J. L. 1998. Epiphytic orchids in eastern subtropical South America. Proceedings of the $15^{\text {th }}$ World Orchid Conference. Turriers, Naturalia Publications, Rio de Janeiro. 494p.

Waechter, J. L. 2002. Padrões geográficos na flora atual do Rio Grande do Sul. Ciência \& Ambiente 24: 93-108.

Warming, E. 1973. Lagoa Santa. In: Warming, E. \& Ferri, M. G. (eds.). Lagoa Santa - a vegetação de cerrados brasileiros. Edusp/ Itatiaia, São Paulo/Belo Horizonte. Pp.1-284.

WWF. 2005. Biomas brasileiros. http:// www.wwf.org.br/natureza_brasileira/ biomas/index.cfm. Acesso em 03/2008. 